\title{
Vitamin E and Bone Structural Changes: An Evidence-Based Review
}

\author{
Isa Naina Mohamed, Boekhtiar Borhanuddin, \\ Ahmad Nazrun Shuid, and Nur Farhana Mohd Fozi
}

Pharmacoepidemiology and Drug Safety Unit, Department of Pharmacology, Faculty of Medicine, Universiti Kebangsaan Malaysia (The National University of Malaysia), Jalan Raja Muda Abdul Aziz, 50300 Kuala Lumpur, Malaysia

Correspondence should be addressed to Nur Farhana Mohd Fozi, nurfarhana_ukm@yahoo.com

Received 5 June 2012; Accepted 7 September 2012

Academic Editor: Srijit Das

Copyright ( $) 2012$ Isa Naina Mohamed et al. This is an open access article distributed under the Creative Commons Attribution License, which permits unrestricted use, distribution, and reproduction in any medium, provided the original work is properly cited.

Purpose. This paper explores the effects of vitamin E on bone structural changes. Methods. A systematic review of the literature was conducted to identify relevant studies about vitamin $\mathrm{E}$ and osteoporosis/bone structural changes. A comprehensive search in Medline and CINAHL for relevant studies published between the years 1946 and 2012 was conducted. The main inclusion criteria were published in English, studies had to report the association or effect of vitamin E and osteoporosis-related bone changes, and the osteoporosis-related bone changes should be related to lifestyle variables, aging, or experimentally-induced conditions. Results. The literature search identified 561 potentially relevant articles, whereby 11 studies met the inclusion criteria. There were three human epidemiological studies and eight animal experimental studies included in this paper. Four animal studies reported positive bone structural changes with vitamin E supplementation. The rest of the studies had negative changes or no effect. Studies with positive changes reported better effects with tocotrienol vitamin $\mathrm{E}$ isomer supplementation. Conclusions. This evidence-based review underscores the potential of vitamin $\mathrm{E}$ being used for osteoporosis. The effect of one of the vitamin $\mathrm{E}$ isomers, tocotrienols, on bone structural changes warrants further exploration. Controlled human observational studies should be conducted to provide stronger evidence.

\section{Introduction}

Osteoporosis is a progressive medical condition in which bone density slowly decreases with advancing age. Osteoporosis is often called "silent" because bone loss occurs without symptoms. An estimated 10 million men and women in the United States have osteoporosis and a further 34 million are at risk [1]. Bone is a highly vascularized connective tissue that contains haematopoietic bone marrow, calcium, and phosphate [2]. Calcium is the most abundant mineral found in bone with approximately $98 \%$ of human body calcium stored in bone. There are two principal cell types in bone: osteoclast and osteoblast. A balance between bone formation by osteoblasts and bone resorption by osteoclasts is needed for bone remodeling and bone development [3]. Osteoporosis is defined as a bone mineral density (BMD) that lies 2.5 standard deviations or more below the average value for young healthy women. Osteoporosis is a progressive systematic skeletal disorder characterized by low bone mineral density (BMD), deterioration of microarchitecture of bone tissue, and susceptibility to fracture caused by bone resorption $[4,5]$. Osteoclast cells have the ability to release free radicals, such as reactive oxygen species (ROS) that will destroy calcified bone tissue and therefore play an integral part in bone remodeling when coupled with osteoblast cells [6-10]. Recent epidemiological studies have also indicated a relationship between oxidative stress and osteoporosis [11, 12].

Vitamin E occurs naturally in eight isoforms: $\alpha, \beta, \gamma$, and $\delta$ isomers of tocopherols and tocotrienols. Each isomer of vitamin $\mathrm{E}$ consists of an aromatic chromanol ring and a side chain. Tocotrienols possess an unsaturated farnesyl (isoprenoid) side chain compared to tocopherols, which have a saturated phytyl side chain [13]. The unsaturated 
side chains of tocotrienols allow them to penetrate more efficiently into the membrane lipid bilayer. Vitamin E has good antioxidant activity, which varies among the different isomers [14]. Vitamin $\mathrm{E}$ is a strong antioxidant that plays a vital role in the endogenous defense against peroxidation of membrane lipids [15]. Tocopherols are abundant in polyunsaturated vegetable oils and in the germ of cereal seed [16]. Tocotrienols are abundant in palm oil, cereal grains, and rice bran [17]. Commercial availability of vitamin $\mathrm{E}$ is mostly in the form of $\alpha$-tocopherol, which is taken as an antioxidant supplement [18]. $\alpha$-Tocopherol has the highest biological activity and is the most abundant form of vitamin $\mathrm{E}$ in human tissues and serum $[19,20]$ as it is selectively retained in the body $[21,22]$. Palm olein (refined, deodorized, and bleached palm cooking oil) contains $196 \mathrm{ppm} \alpha$-tocopherol, $201 \mathrm{ppm} \alpha$-tocotrienol, $372 \mathrm{ppm} \beta$-tocotrienol, and $96 \mathrm{ppm}$ $\gamma$-tocotrienol [23]. As described, tocotrienols are found abundant in palm oil and have been reported to be better antioxidants compared to tocopherols [24, 25].

Evidence of vitamin E having a beneficial effect against osteoporosis has been actively researched only recently. Vitamin $\mathrm{E}$ has been reported to play a role in increasing bone density. According to researcher NS Ahmad and colleagues in their research on nicotine-treated rats, vitamin $\mathrm{E}$ increases the trabecular bone (a spongy-looking bone), prevents bone calcium loss by neutralizing antioxidants, and decreases bone loss of calcium in rats without ovaries [26]. Vitamin E supplementation was able to protect bones from oxidative damage by scavenging free radicals $[27,28]$ and was able to maintain bone matrix trophysm and stimulate trabecular bone formation $[29,30]$. Previous studies reported that vitamin E supplementation protects against bone loss and damage caused by oxidative stress, which is induced by sex hormones deficiency $[31,32]$ or oxygen-derived free radicals [33-35]. Postovariectomised rats have similar bone changes to those of postmenopausal women [36]. Vitamin E supplementation protects against bone loss and restores bone strength in the aged mouse [37] and ovariectomized rat $[32,38]$ - both are accepted osteopenic models.

Nicotine has been reported to increase proinflammatory mediators (through oxidative stress), resulting in bone loss and reduced bone mechanical strength (through inhibition of osteoblasts) in rats [39-41]. In humans, smoking is a recognized risk factor for osteoporosis [42, 43]. Recent studies utilising nicotine-induced rat model have reported that vitamin $\mathrm{E}$ was able to prevent the increment of boneresorbing cytokines [26] and reverse the damage on bone histomorphometry [44].

Calcium plays an integral part in bone metabolism and remodeling [45]. A vitamin E-deficient diet will result in bone damage, probably due to impaired calcium absorption $[46,47]$ that leads to a state of calcium deficiency [48] and increased free radical activity [49].

Elevated levels of bone-resorbing cytokines, mainly interleukin 1 and 6 (IL-1 and IL-6), are known to be associated with accelerated bone resorption after menopause [50-52]. IL-1 is secreted by monocytes in an estrogen deficiency state that will induce osteoblast to secrete IL-6 [50]. IL-6 will stimulate osteoclast proliferation and subsequently increase bone resorption $[52,53]$. Vitamin E, especially tocotrienol that was reported to be more potent compared to tocopherol, was reported to be able to prevent the rise of serum IL- 1 and adverse effects of free radicals on trabecular bone structure $[33,34]$.

The increasing number of studies, especially in the past decade, which focused on the role of vitamin $\mathrm{E}$ in the prevention or treatment for osteoporosis warrants a review. The aim of this evidence-based review is therefore to explore original research articles in order to determine the effects of vitamin $\mathrm{E}$ on bone structural changes.

\section{Methods}

2.1. Literature Review. A systematic review of the literature was conducted to identify relevant studies about vitamin $\mathrm{E}$ and osteoporosis/bone structural changes. To conduct a comprehensive search of health science journals, we used Medline via Ovid Medline (published between 1946 and March 2012) and CINAHL via Ebscohost (published between 1946 and 2012). The search strategy involved a combination of the following two sets of key words (1) vitamin e OR vit* e OR tocotrienol OR tocopherol; (2) bone* OR bone metabolis* OR bone mineral* OR osteoblast* OR osteoclast* OR osteoporo* OR osteopen* OR osteogen*.

2.2. Selection of Research Articles. The results were limited to studies that were published in English language that included abstracts. To be included, studies had to (1) report the association or effect of vitamin $\mathrm{E}$ and osteoporosisrelated bone changes and (2) the osteoporosis-related bone changes should be related to lifestyle variables, aging, or experimentally-induced conditions. Papers were excluded if the studies were related to (1) osteoporosis that is related to other pathological changes; (2) reviews, news, letter, editorials, or case studies; (3) bone fracture healing; or (4) fetal bone or bone marrow formation.

2.3. Data Extraction and Management. We selected papers to be included in the review in three phases. First, we excluded any paper that did not match the inclusion criteria based solely on the title. Second, we screened all the abstracts of the remaining papers and then excluded a second group of papers that did not meet our inclusion criteria. Lastly, we read the remaining papers from the second phase to exclude any paper that did not meet our inclusion criteria.

After the initial screening of the titles and abstracts, duplicates were removed and the remaining papers were again screened by at least two reviewers. The inclusion of the full papers into the review had to be agreed by at least two reviewers before the data extraction phase. Any discrepancies were resolved through discussion between the reviewers. Data extraction was performed independently and in a standardized manner with the use of a data collection form. We recorded the following data from the studies: (1) the type of study and vitamin E analog studied; (2) a brief description of the sample/population of the study; (3) a brief 
description of the methods used in the study; (4) the brief description of the results of the study; (5) our comments and conclusion of the study.

\section{Results}

3.1. Search Results. The literature searches identified 561 potentially relevant articles. Two reviewers independently assessed all articles for inclusion or exclusion based on the title and abstract. A total of 42 articles were retrieved for further assessment and data extraction. Fifteen of these articles were excluded because they did not focus on primary studies $(n=8)$ or because they were not related with vitamin $\mathrm{E}$ and osteoporosis.

Differences of opinion between the reviewers regarding the inclusion or exclusion of the full articles were resolved by discussion. 16 articles from the remaining 27 articles were excluded based on the inclusion and exclusion criteria. A total of 11 articles were included for the purpose of this review. A flow chart of the selection and paper process, including reasons for exclusion, is shown in Figure 1.

3.2. Study Characteristics. The summary of the characteristics of all studies is displayed in Table 1 (animal studies) and Table 2 (human studies). All studies were conducted after the year 2000 with the majority conducted in the past five years. There were three human studies and eight animal studies that used rats as their study model. Although only three of human studies were included in this paper, each of these studies by Macdonald et al. [60], Maggio et al. [59], and Wolf et al. [61] had a large population sample size of at least 150 women. The study by Wolf et al. [61] had a sample of 11,068 participants and hence provided ample power and confidence for the generated results. All human studies involved women only, with bone mineral density (BMD) measurement being the main measured outcome, thus providing a highly reliable indicator for osteoporosis. Studies by Macdonald HM and Wolf RL collected information on vitamin E consumption through the use of questionnaires. It was not mentioned whether the questionnaires were validated. Vitamin E consumption from diet and supplements were then correlated with BMD measurements to determine the effect of vitamin E consumption on BMD. Maggio compared the plasma vitamin E level of osteoporotic participants versus nonosteoporotic participants to determine if there are any differences in vitamin $\mathrm{E}$ levels of women suffering from osteoporosis.

One animal study used Winstar rats with the remaining seven studies using Sprague-Dawley rats. Rats were of various ages, with the number of rats for each study (ranging from 24 to 96 rats) kept to a minimal number due to animal ethics requirements. Seven studies [32, 34, 44, 54, 55, 57, 58] assessed trabecular and cortical bone structure using bone histomorphometry analysis of the rat bones after treatment with vitamin $E$. Three studies $[32,55,56]$ conducted various blood biochemical tests, three studies $[32,54,56]$ conducted BMD scans, one study [57] conducted a biomechanical test to assess bone strength, and one study [56] used Micro-CT to assess trabecular and cortical bone structure. All animal studies were conducted using experimental design which compared the outcome of vitamin $\mathrm{E}$ treated groups (tocopherol, tocotrienol, or combination of various amounts) with control or sham group.

\section{Effects of Vitamin E on Osteoporosis for Human Epidemiological Study}

There were three human studies included in this paper. The study by Maggio et al. [59], was conducted to determine whether antioxidant defense (including vitamin E) decreased in osteoporotic elderly women by measuring antioxidant plasma levels of participants. There were a total of 150 women participants, whereby 75 women with diagnosed osteoporosis were compared with 75 women with normal BMD (control). The sample characteristics were as follows:mean age: 70.4 years; mean BMI: 25.3; mean years since menopause: 22.8 years. The reported results indicated that the mean plasma levels of vitamin $\mathrm{E}$ were significantly lower in osteoporotic women, if compared to control participants $(P<0.001)$. The authors suggested the need of further research to determine the relevance and the mechanism of action between low levels of vitamin $\mathrm{E}$ and osteoporosis.

Macdonald et al. [60], conducted a large longitudinal study with 891 women participants to determine the association between antioxidants (including vitamin E) intake and BMD. The characteristics of study participants were as follows: mean age: 53.9 years; mean BMI: 26.1; total vitamin E intake: $13.3 \mathrm{mg}$. Macdonald concluded that there was no evidence of any association between nutrient intake and BMD change. Total vitamin $\mathrm{E}$ intake (dietary plus supplementation) correlated positively with BMD, but was not significant. Dietary vitamin E intake was significantly correlated with BMD $(P<0.01)$, but was negatively correlated.

The largest human epidemiological study included in this review was conducted by Wolf et al. [61], involving 11,068 women participants (mean age: 63.2 years; mean BMI: 28.3; mean total vitamin E intake: $28.9 \mathrm{mg}$ ). Due to the high number of participants, the regression analysis done in this study had significant power, with multiple covariate adjustments performed. Wolf reported positive correlation between dietary vitamin $\mathrm{E}$ and femoral neck BMD when adjusted for age only. After adjusting for multiple BMD related covariates, no significant association was found between vitamin $\mathrm{E}$ consumption and all BMD parameters.

\section{Effects of Vitamin E on Osteoporosis for Human Animal Study}

A total of 10 animal studies were included in this paper. Ahmed et al. have two studies included in this paper. Shuid et al. used bone histomorphometry and biomechanical strength model, whereby $\gamma$-tocotrienol supplementation produced greater trabecular volume and number compared to control rats and rats supplemented with $\alpha$-tocopherol [57]. Using Ferric nitrilotriacetate (FeNTA) to induce diabetes in rats and subsequently bone damage, N.S. Ahmad et al. reported 


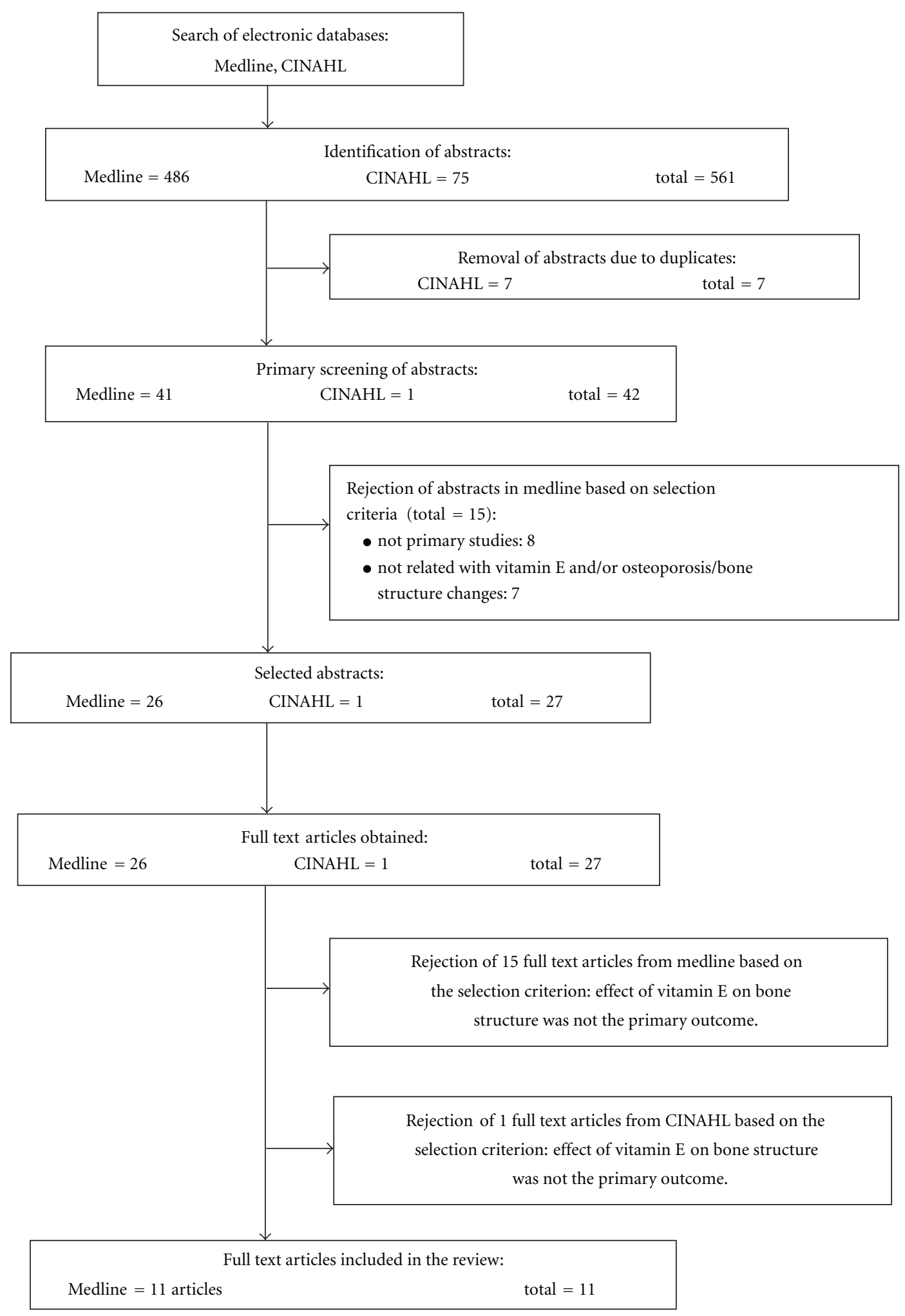

FIgURE 1: Flow chart to show the selection process of the articles in this review. 


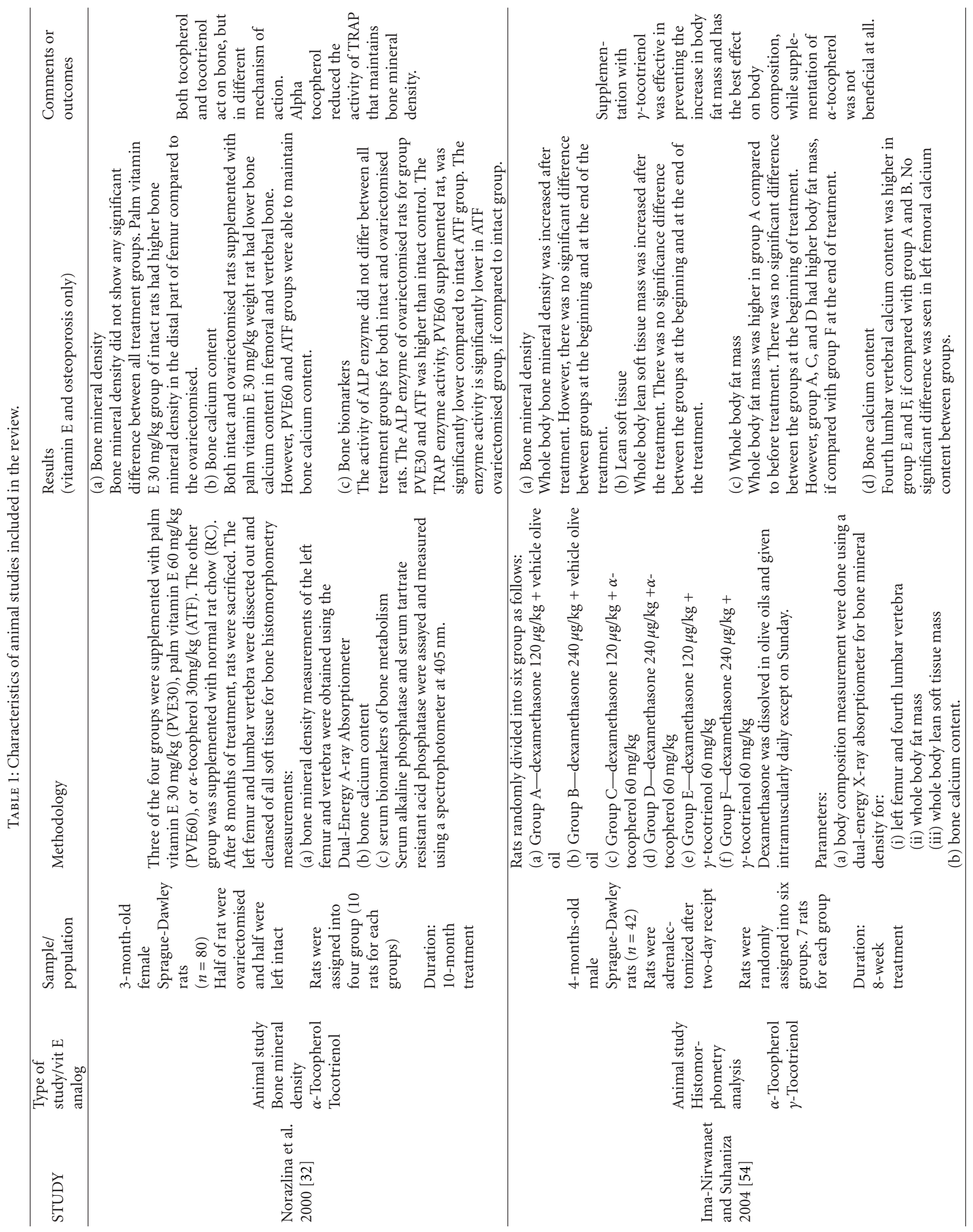




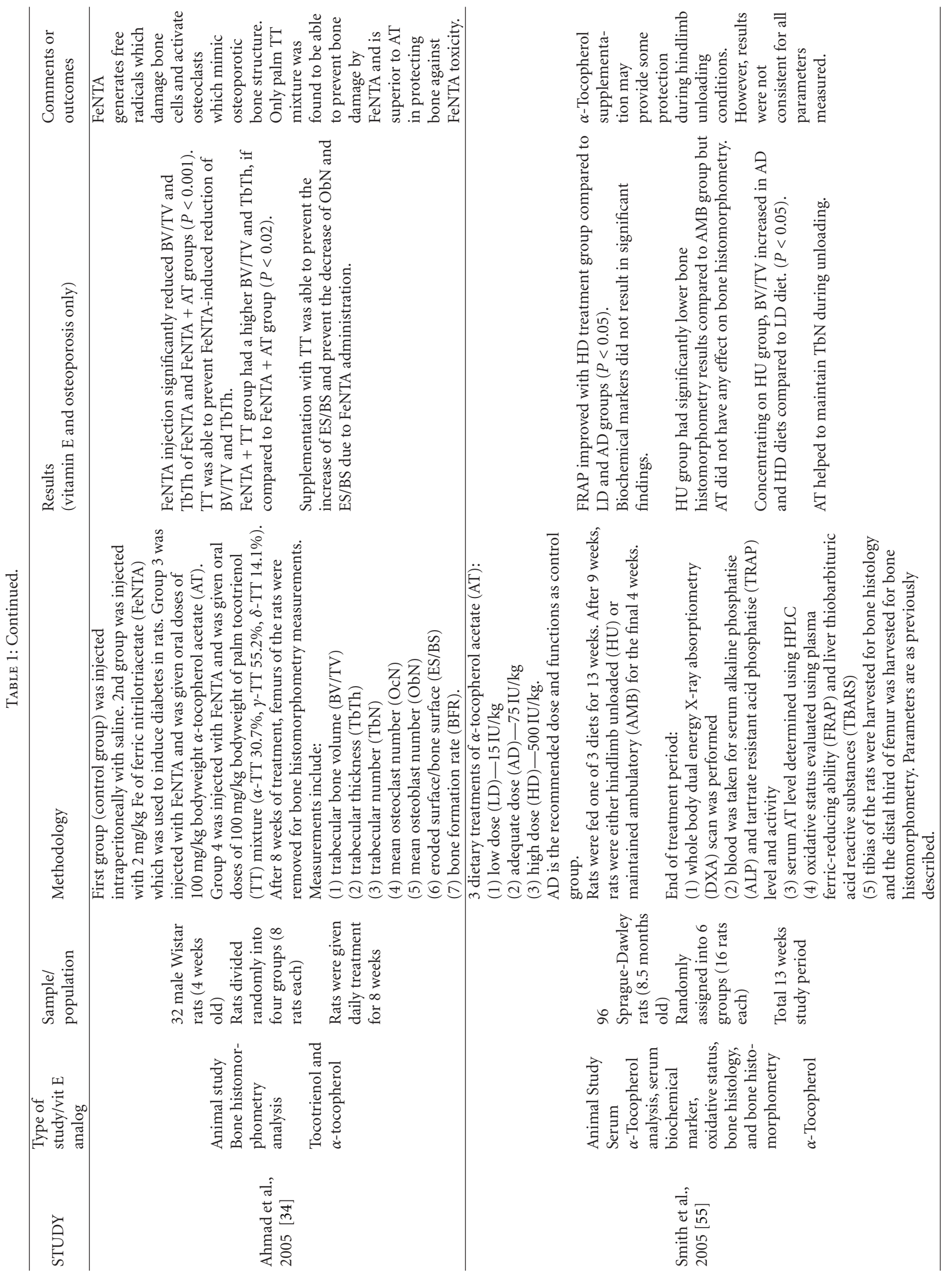




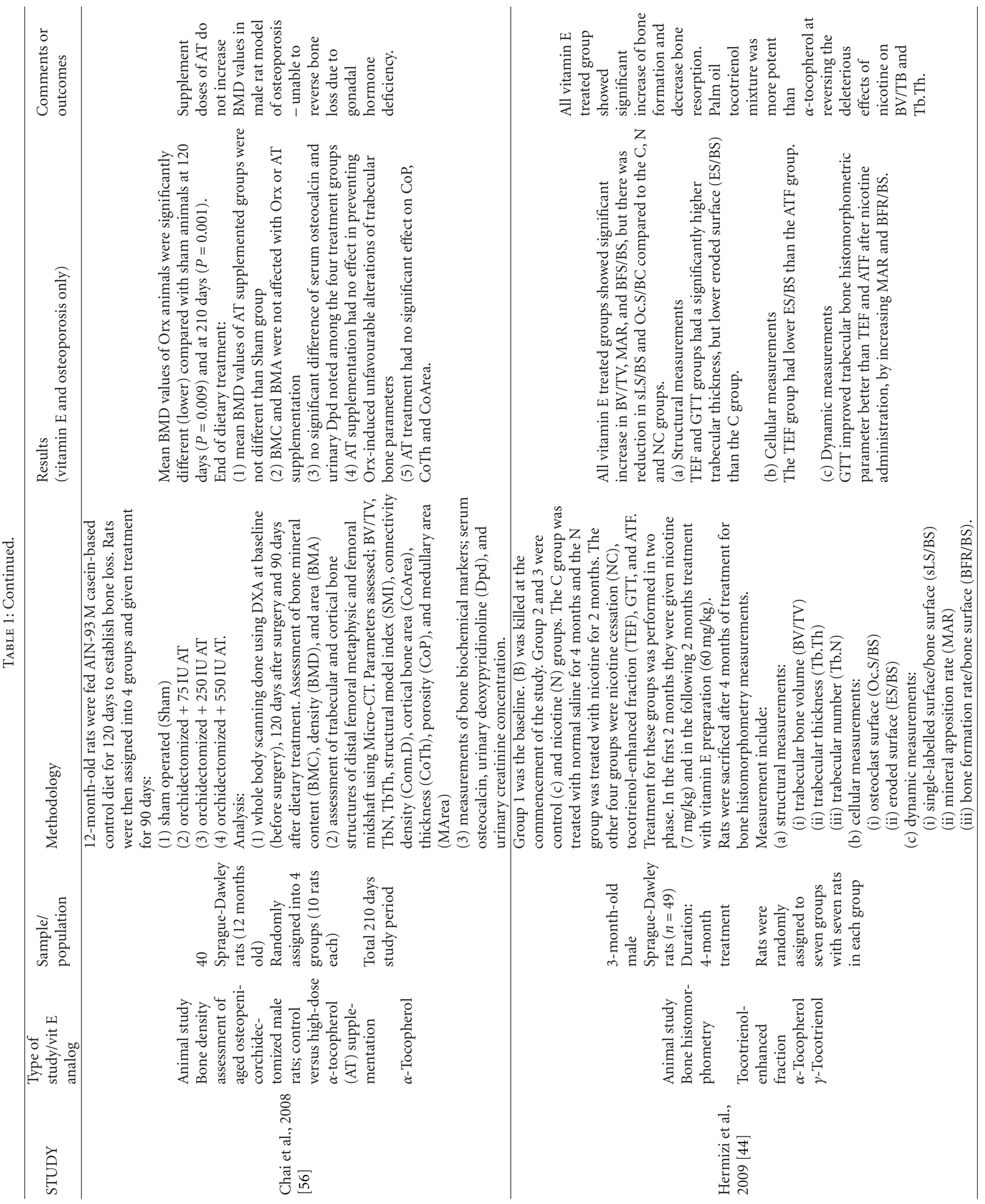




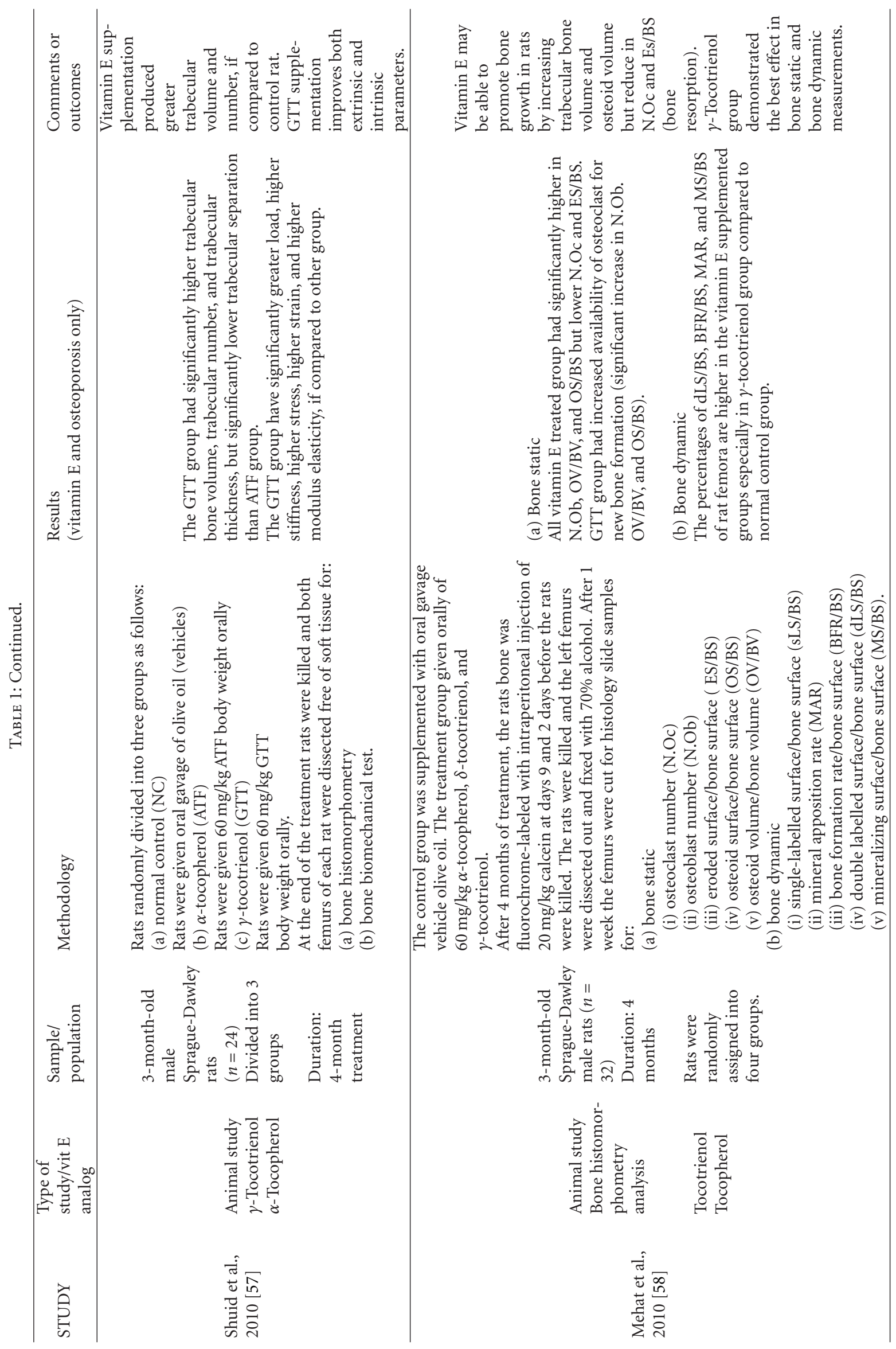




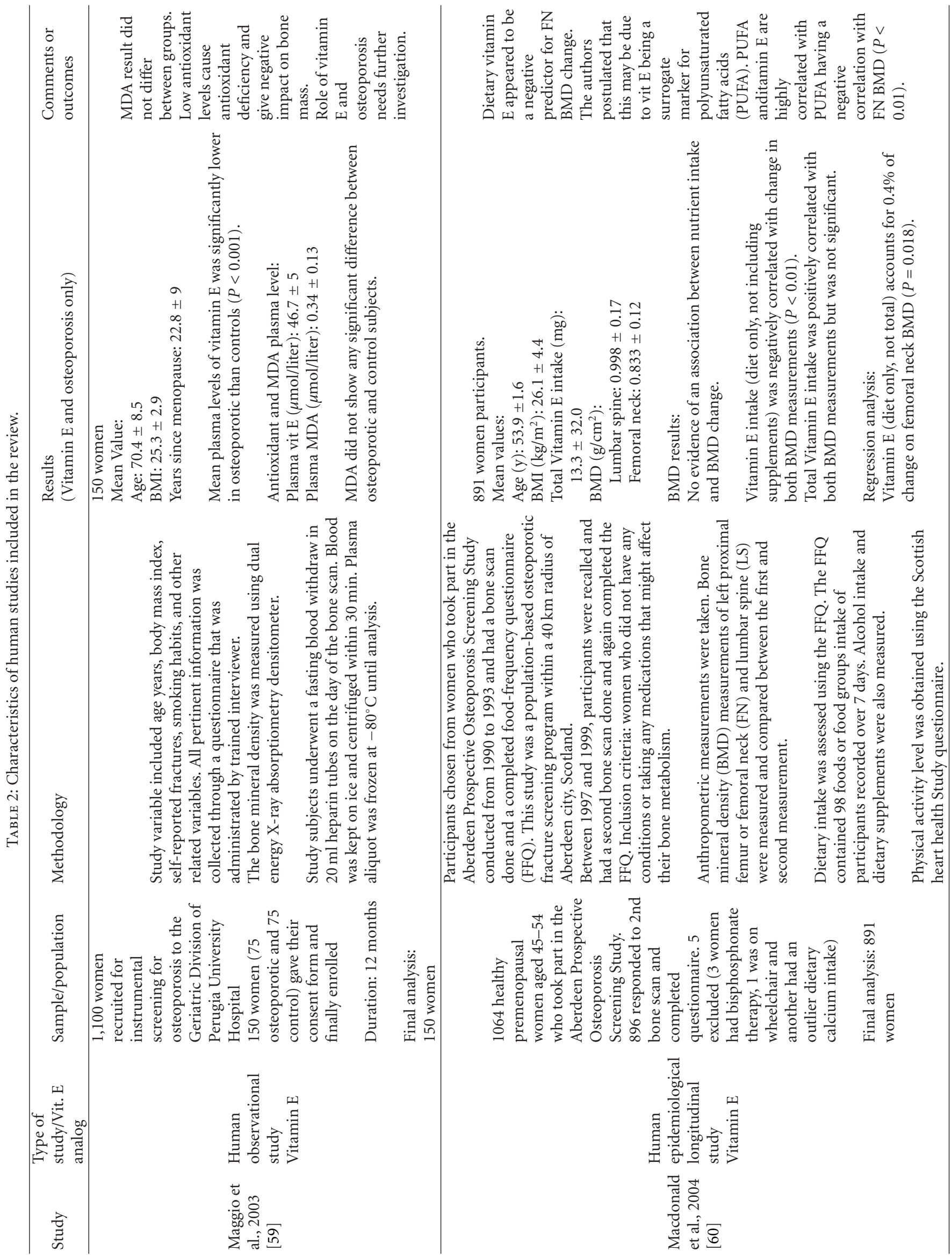




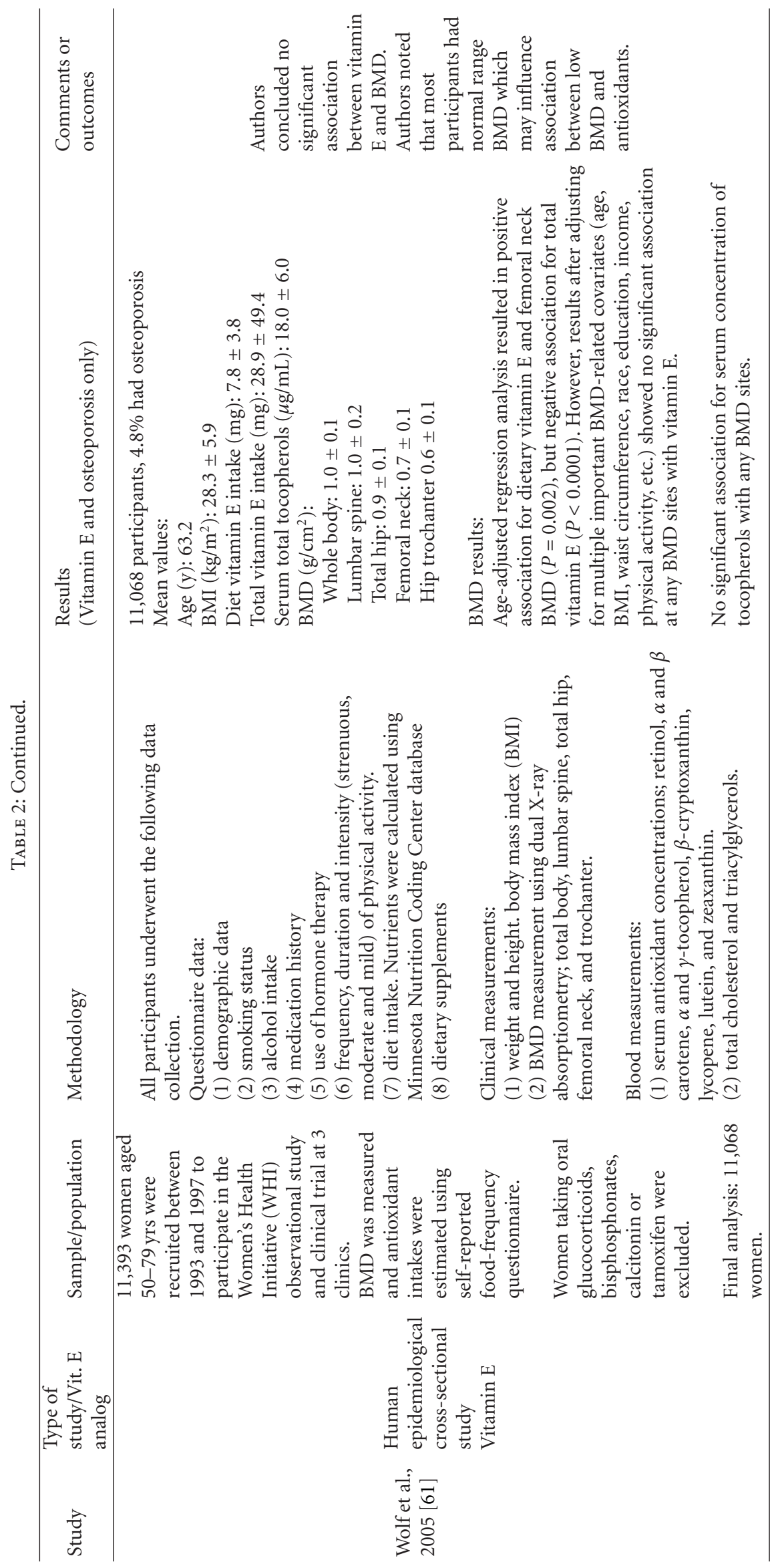


that palm tocotrienol mixture (consisting of $\alpha$-TT 30.7\%, $\gamma$ TT $55.2 \%, \delta$-TT $14.1 \%$ ) supplementation was able to prevent bone damage [34]. Palm tocotrienol was also reported to have superior protection properties compared to $\alpha$ tocopherol.

Ima-Nirwana and Suhaniza reported that supplementation with $\gamma$-tocotrienol or palm vitamin E successfully prevented osteoporosis in adrenalectomised rats replaced with dexamethasone, if compared to control and rats supplemented with $\alpha$-tocopherol [54]. Norazlina et al. in their study (published in the year 2000) compared ovariectomized rats and nonovariectomized rats supplemented with palm vitamin $\mathrm{E}$ or $\alpha$-tocopherol. There was no significant change between the treatment groups, in terms of BMD obtained from histomorphometry [32]. Hermizi et al., in a 2009 study using nicotine-induced rat bone damage model, reported that vitamin $\mathrm{E}$ was able to reverse the effect of nicotine damage on bone structure and noted $\gamma$-tocotrienol supplementation caused significantly higher mineral appositional rate and bone formation rate, if compared to tocotrienolenhanced fraction and $\alpha$-tocopherol treated-group [44].

In a recent study by Mehat et al. 2010, rats supplemented with oral vitamin $\mathrm{E}$ for four months had increased trabecular and osteoid bone volume, and reduction of bone resorption parameters [58]. $\gamma$-tocotrienol supplementation produced the best bone measurements improvement, if compared to $\delta$-tocotrienol and $\alpha$-tocopherol [58]. Smith et al. reported that $\alpha$-tocopherol did not have any effects on bone histomorphometry parameters when supplemented at varying doses in hindlimb unloaded rats, if compared to ambulatory rats [55]. Chai et al. reported similar negative findings on varying doses of $\alpha$-tocopherol supplementation in male orchidectomized rats [56]. No significant BMD parameters were observed and Chai et al. concluded that $\alpha$-tocopherol supplementation was not able to reverse bone loss due to gonadal hormone deficiency [56].

\section{Discussion}

This paper has mixed main findings. There was strong evidence of the benefit of vitamin $\mathrm{E}$ supplementation in rats, whereby positive changes in bone structure were demonstrated for four of the eight animal studies [34, 44, 57, 58]. Three out of the four remaining animal studies $[32,54$, 57] reported positive findings of vitamin $E$ supplementation for biochemical results, although not for bone structure parameters. Only Chai et al. reported no positive finding for vitamin E supplementation in rats. Rat animal model has become acceptable for human bone studies due to the similar mechanism of control gain and loss of bone mass compared to humans. All the three human studies reported no association between vitamin $\mathrm{E}$ consumption and BMD changes [59-61].

Nicotine causes an increase of proinflammatory mediators (oxidative stress) that results in bone loss and reduction of bone mechanical strength (through the inhibition of osteoblasts) in rats [39-41]. In humans, smoking is a recognized risk factor for osteoporosis [42, 43]. Hermizi et al. reported that vitamin $\mathrm{E}$ treated rats were able to recover from nicotine-induced bone damage, where tocotrienol mixture was more potent compared to tocopherol [44]. Postulated mechanisms were antioxidative effects of vitamin $\mathrm{E}$ and the reduction of free radicals.

Five studies denoted a differing effect towards bone structure between vitamin $\mathrm{E}$ isomers, mainly between tocotrienol and tocopherol $[34,44,54,57,58]$. It has been postulated that tocotrienol has better activity compared to $\alpha$-tocopherol due to the higher mobility of polyenoic lipids of tocotrienol in the membrane bilayer and therefore is more mobile and less restricted in its interaction with lipid radicals in the membranes [24]. Both of Ahmad et al. studies employed histomorphometry technique to view changes of rat bone structures [34, 57]. In their 2005 study, vitamin E treated rats were able to maintain their bone structure, resisting FeNTA-induced bone damage. Ahmad et al. supplemented $\alpha$-tocopherol and tocotrienol mixture on different groups of rats and noted that only rats treated with tocotrienol mixture was able to effectively resist bone damage induced by FeNTA [34]. In a recent study by shuid et al. (2010), they reported that vitamin $\mathrm{E}$ supplementation had positive anabolic changes on bone structure (i.e., increased trabecular volume and number) compared to control rats [57]. Both studies concluded that improvement was superior among tocotrienol treated-rats compared to $\alpha$-tocopherol-treated rats. Mehat et al. conducted a study specifically to determine bone structural changes of rats supplemented with different isomers of vitamin $\mathrm{E}(\alpha$-tocopherol versus $\delta$-tocotrienol versus $\gamma$-tocotrienol) [58]. Bone histomorphometry analysis revealed that all isoforms promote bone growth in rats with $\gamma$-tocotrienol, producing the best effect in both static and dynamic measurements in bones. Mehat et al. did not suggest a mechanism or reason to explain the differences observed between the isomers of vitamin $\mathrm{E}$.

Three out of four remaining animal studies reported positive results for positive biochemical changes, but without any significant bone structural changes. Among the biochemical changes reported were the increase of bone calcium content $[32,54]$, the reduction in oxidative status measured using plasma ferric-reducing ability [55], the improvement of serum alkaline phosphatase (ALP) and the reduction of serum tartrate-resistant acid phosphatase (TRAP) [32], and the improvement of whole body fat mass [54]. Serum ALP is a measurement of osteoblastic activity, while serum TRAP is specific for osteoclastic activity [62]. Ima-Nirwana and Suhaniza reported $\gamma$-tocotrienol-treated rats had the best effect on body composition and postulated that the effect could be due to tocotrienol's antioxidant effect or improvement of calcium transport and utilization [54]. Improvements of either bone structure parameters or biochemical parameters were mostly attributed to vitamin $\mathrm{E}$ through its antioxidant effects, free-radical scavenging capability, protection from cellular lipid peroxidation, improvement of calcium transport and utilization, and the suppression of bone resorbing cytokines, IL-1 and IL-6.

Maggio et al. in his study confirmed that plasma concentration of vitamin $\mathrm{E}$ is lower in osteoporotic women when compared to nonosteoporotic women [59]. However, all the 
three human epidemiological studies in this paper reported no significant association between vitamin E consumption and BMD improvement [59-61]. The largest study, conducted by Wolf et al. with 11,068 participants, had positive age-adjusted regression analysis for dietary Vitamin $\mathrm{E}$ and femoral neck BMD $(P=0.002)$ association, but negative association for total vitamin $\mathrm{E}(P<0.0001)$ [61]. Due to the large number of participants and sufficient statistical power, Wolf et al. were able to perform regression analysis that adjusted for more than 50 important BMD-related covariates. However, no significant association was reported. Wolf et al. offered several possible explanations of the mixed results: the variety in exposure measurement, outcome measurement and BMD site measurement, and other confounding factors. Among the three human epidemiological reviews, Macdonald et al. had reported negative correlation between dietary vitamin $\mathrm{E}$ and BMD measurements $(P<$ 0.01 ), although this association was not persistent for the total vitamin E consumption, suggesting a possible opposite outcome if vitamin $\mathrm{E}$ was taken in larger doses [60].

Comparing human epidemiological studies with animal studies outcome is often difficult, as translation of results from animals to humans is hampered by various differences. The mechanisms of which vitamin E exerts its effects could be different in humans. Controlling environmental factors, food intake, and confounding factors is often possible in animal studies, but impossible in human epidemiological studies. Interactions between vitamin $\mathrm{E}$ and other antioxidants, nutrients, chemicals, and other food sources have to be explored. The stark differences, with almost opposite outcomes between animal studies and human epidemiological studies in this review, are therefore not surprising. All human epidemiological studies in this review reported consumption of vitamin $\mathrm{E}$ as a study variable, but the distinction between the different vitamin $\mathrm{E}$ isomers (tocopherols and tocotrienols) was not made. The majority of animal studies actually reported a positive outcome, or at least a superior effect for tocotrienols compared to tocopherols. This could offer a possible explanation for the discrepancy of the study outcomes. We were unable to provide a definitive answer whether vitamin $\mathrm{E}$ has any significant effects on bone structure, either positive or negative. The outcomes of studies included in this review were mixed. Half of the animal studies had positive changes on bone structure, whereas none of the human epidemiological studies had positive effects. Tocotrienols seemed to exert superior effects on bone structure and further studies concentrating on this isomer would need to be explored. Empirical human case-control studies or randomized control trials with vitamin $\mathrm{E}$ or its isomer derivatives would help ascertain the effect of vitamin E on bone structure.

Strength and Limitation of This Review. The research on the effects of vitamin $\mathrm{E}$ on osteoporosis is a promising field with important findings published in the last decade and a critical review is therefore highly relevant. Our search identified 11 research articles that were included in this paper and we believe that this is the first critical review on this subject matter that focuses on vitamin $\mathrm{E}$ and bone structural changes. We have also included both animal studies and human studies in this review, providing a better overview of the most recent and reliable evidence available.

This review has several limitations. Many studies did not differentiate the many isomers of vitamin $\mathrm{E}$ in their study. Due to the difference in the effect and activity of the various isomers, generalization of results and outcomes for the effect of vitamin $\mathrm{E}$ had to be further scrutinized. Many of the original research articles included in this review had other parameters included, especially blood biochemical parameters. The human studies focused on all antioxidants and mostly concentrating on vitamin $\mathrm{C}$, instead of vitamin E. Nevertheless, results were thoroughly screened to avoid misrepresentation of the results of other antioxidants. All the human studies included in the review were epidemiological in nature, which presents with its own inherent weaknesses. Despite this limitation, the number of participants for each human epidemiological study was large. For example, the study by Wolf et al. had over 11,000 participants, providing ample statistical power and the inclusion of various adjusted covariates in the multiple-regression analysis.

Recommendations. Based on the heterogeneity of the study methods, especially among the animal studies, it is crucial that future studies use a standardized protocol to examine the effect of vitamin $\mathrm{E}$ various isomers on a consensual gold-standard parameter that assesses the osteoporotic state. Besides that, more effort should be made in designing controlled human observational studies that will help in reducing the numbers of potential confounders in the analysis of the result. These measures will ensure that proper meta-analysis could be conducted in the future to give us a clearer picture about the actual effect of vitamin $\mathrm{E}$ on osteoporosis.

\section{Conclusion}

This evidence-based review underscores the potential of vitamin E being used for osteoporosis, and sought to look into vitamin $\mathrm{E}$ and bone structure changes. The effect of vitamin $\mathrm{E}$ isomers, especially tocotrienols, on bone structural changes warrants further exploration. Additionally, controlled human observational studies should be conducted to provide stronger evidence. Due to the mix outcomes of the studies included in this paper, it is currently premature to state that vitamin $\mathrm{E}$ has positive, negative, or no effect on bone structure, at least until more studies are conducted.

\section{References}

[1] National Osteoporosis Foundation, 2012, http://www.nof.org/ aboutosteoporosis/bonebasics/whybonehealth.

[2] D. A. Stevens and G. R. Williams, "Hormone regulation of chondrocyte differentiation and endochondral bone formation," Molecular and Cellular Endocrinology, vol. 151, no. 1-2, pp. 195-204, 1999.

[3] S. L. Teitelbaum, "Bone resorption by osteoclasts," Science, vol. 289, no. 5484, pp. 1504-1508, 2000. 
[4] S. Roux and P. Orcel, "Bone loss: factors that regulate osteoclast differentiation," Arthritis Research \& Therapy, vol. 2, no. 6, pp. 451-456, 2000.

[5] World Health Organisation, 2012, http://www.who.int/chp/ topics/Osteoporosis.pdf/.

[6] S. Muthusami, I. Ramachandran, B. Muthusamy et al., "Ovariectomy induces oxidative stress and impairs bone antioxidant system in adult rats," Clinica Chimica Acta, vol. 360, no. 1-2, pp. 81-86, 2005.

[7] N. Suda, I. Morita, T. Kuroda, and S. I. Murota, "Participation of oxidative stress in the process of osteoclast differentiation," Biochimica et Biophysica Acta, vol. 1157, no. 3, pp. 318-323, 1993.

[8] S. Yang, P. Madyastha, S. Bingel, W. Ries, and L. Key, "A new superoxide-generating oxidase in murine osteoclasts," The Journal of Biological Chemistry, vol. 276, no. 8, pp. 54525458, 2001.

[9] Z. Khalkhali-Ellis, P. Collin-Osdoby, L. Li, M. L. Brandi, and P. Osdoby, "A human homolog of the $150 \mathrm{kD}$ avian osteoclast membrane antigen related to superoxide dismutase and essential for bone resorption is induced by developmental agents and opposed by estrogen in FLG 29.1 cells," Calcified Tissue International, vol. 60, no. 2, pp. 187-193, 1997.

[10] R. J. Van't Hof and S. H. Ralston, "Nitric oxide and bone," Immunology, vol. 103, no. 3, pp. 255-261, 2001.

[11] S. Basu, K. Michaëlsson, H. Olofsson, S. Johansson, and H. Melhus, "Association between oxidative stress and bone mineral density," Biochemical and Biophysical Research Communications, vol. 288, no. 1, pp. 275-279, 2001.

[12] S. Yalin, S. Bagis, G. Polat et al., "Is there a role of free oxygen radicals in primary male osteoporosis?" Clinical and Experimental Rheumatology, vol. 23, no. 5, pp. 689-692, 2005.

[13] E. Serbinova, V. Kagan, D. Han, and L. Packer, "Free radical recycling and intramembrane mobility in the antioxidant properties of $\alpha$-tocopherol and alpha-tocotrienol," Free Radical Biology and Medicine, vol. 10, no. 5, pp. 263-275, 1991.

[14] A. Kamal-Eldin and L. A. Appelqvist, "The chemistry and antioxidant properties of tocopherols and tocotrienols," Lipids, vol. 31, no. 7, pp. 671-701, 1996.

[15] L. Packer, S. U. Weber, and G. Rimbach, "Molecular aspects of $\alpha$-tocotrienol antioxidant action and cell signalling," Journal of Nutrition, vol. 131, no. 2, pp. 369S-373S, 2001.

[16] Y. Yoshida, E. Niki, and N. Noguchi, "Comparative study on the action of tocopherols and tocotrienols as antioxidant: chemical and physical effects," Chemistry and Physics of Lipids, vol. 123, no. 1, pp. 63-75, 2003.

[17] C. K. Sen, S. Khanna, S. Roy, and L. Packer, "Molecular basis of vitamin E action: tocotrienol potently inhibits glutamateinduced pp60c-src kinase activation and death of HT4 neuronal cells," The Journal of Biological Chemistry, vol. 275, no. 17, pp. 13049-13055, 2000.

[18] A. Azzi and A. Stocker, "Vitamin E: non-antioxidant roles," Progress in Lipid Research, vol. 39, no. 3, pp. 231-255, 2000.

[19] M. G. Traber, "Vitamin E regulatory mechanisms," Annual Review of Nutrition, vol. 27, pp. 347-362, 2007.

[20] K. M. Fairfield and R. H. Fletcher, "Vitamins for chronic disease prevention in adults: scientific review," The Journal of the American Medical Association, vol. 287, no. 23, pp. 31163126, 2002.

[21] M. Arita, Y. Sato, A. Miyata et al., "Human $\alpha$-tocopherol transfer protein: cDNA cloning, expression and chromosomal localization," Biochemical Journal, vol. 306, no. 2, pp. 437-443, 1995.

[22] A. Hosomi, M. Arita, Y. Sato et al., "Affinity for $\alpha$-tocopherol transfer protein as a determinant of the biological activities of vitamin E analogs," FEBS Letters, vol. 409, no. 1, pp. 105-108, 1997.

[23] C. E. Elson, "Tropical oils: nutritional and scientific issues," Critical Reviews in Food Science and Nutrition, vol. 31, no. 1-2, pp. 79-102, 1992.

[24] J. P. Kamat and T. P. A. Devasagayam, "Tocotrienols from palm oil as potent inhibitors of lipid peroxidation and protein oxidation in rat brain mitochondria," Neuroscience Letters, vol. 195, no. 3, pp. 179-182, 1995.

[25] J. P. Kamat, H. D. Sarma, T. R. A. Devasagayam, K. Nesaretnam, and Y. Basiron, "Tocotrienols from palm oil as effective inhibitors of protein oxidation and lipid peroxidation in rat liver microsomes," Molecular and Cellular Biochemistry, vol. 170, no. 1-2, pp. 131-138, 1997.

[26] M. Norazlina, P. L. Lee, H. I. Lukman, A. S. Nazrun, and S. Ima-Nirwana, "Effects of vitamin E supplementation on bone metabolism in nicotine-treated rats," Singapore Medical Journal, vol. 48, no. 3, pp. 195-199, 2007.

[27] A. Azzi, I. Breyer, M. Feher et al., "Specific cellular responses to $\alpha$-tocopherol," Journal of Nutrition, vol. 130, no. 7, pp. 1649$1652,2000$.

[28] R. Ricciarelli, J. M. Zingg, and A. Azzi, "Vitamin E: protective role of a janus molecule," The FASEB Journal, vol. 15, no. 13, pp. 2314-2325, 2001.

[29] M. Passeri and D. Provvedini, "Vitamin E in the physiopathology of the elderly," Acta Vitaminologica et Enzymologica, vol. 5, no. 1, pp. 53-63, 1983.

[30] H. Xu, B. A. Watkins, and M. F. Seifert, "Vitamin E stimulates trabecular bone formation and alters epiphyseal cartilage morphometry," Calcified Tissue International, vol. 57, no. 4, pp. 293-300, 1995.

[31] S. Ima-Nirwana, A. Kiftiah, A. G. Zainal, M. Norazlina, M. T. Gapor, and B. A. K. Khalid, "Palm vitamin E prevents osteoporosis in orchidectomized growing male rats," Natural Product Sciences, vol. 6, no. 4, pp. 155-160, 2000.

[32] M. Norazlina, S. Ima-Nirwana, M. T. Gapor, and B. A. K. Khalid, "Palm vitamin $\mathrm{E}$ is comparable to $\alpha$-tocopherol in maintaining bone mineral density in ovariectomised female rats," Experimental and Clinical Endocrinology and Diabetes, vol. 108, no. 4, pp. 305-310, 2000.

[33] N. S. Ahmad, B. A. K. Khalid, and S. Ima-Nirwana, "Effects of vitamin $\mathrm{E}$ on interleukin-1 in ferric-nitrilotriacetate treated rats," Malaysian Journal Biochemistry and Biology, vol. 9, pp. 43-47, 2004.

[34] N. S. Ahmad, B. A. K. Khalid, D. A. Luke, and S. Ima-Nirwana, "Tocotrienol offers better protection than tocopherol from free radical-induced damage of rat bone," Clinical and Experimental Pharmacology \& Physiology, vol. 32, no. 9, pp. 761-770, 2005.

[35] J. K. Yee and S. Ima-Nirwana, "Palm vitamin E protects against ferric-nitriltrioacetate-induced impairment of bone calcification," Asia Pacific Journal of Pharmacology, vol. 13, no. 1, pp. 35-41, 1998.

[36] D. N. Kalu, C. C. Liu, R. R. Hardin, and B. W. Hollis, "The aged rat model of ovarian hormone deficiency bone loss," Endocrinology, vol. 124, no. 1, pp. 7-16, 1989.

[37] B. H. Arjmandi, S. Juma, A. Beharka, M. S. Bapna, M. Akhter, and S. N. Meydani, "Vitamin E improves bone quality in the 
aged but not in young adult male mice," Journal of Nutritional Biochemistry, vol. 13, no. 9, pp. 543-549, 2002.

[38] B. H. Arjmandi, M. P. Akhter, D. Chakkalakal et al., "Effects of isoflavones, vitamin $\mathrm{E}$, and their combination on bone in an aged rat model of osteopenia," Journal of Bone Mineral Research, vol. 16, article S553, 2001.

[39] P. D. Broulik, J. Rosenkrancová, P. Růžička, R. Sedláček, and I. Kurcová, "The effect of chronic nicotine administration on bone mineral content and bone strength in normal and castrated male rats," Hormone and Metabolic Research, vol. 39, no. 1, pp. 20-24, 2007.

[40] R. G. Cooper and T. Magwere, "Nitric oxide-mediated pathogenesis during nicotine and alcohol consumption," Indian Journal of Physiology and Pharmacology, vol. 52, no. 1, pp. 1118, 2008.

[41] M. A. Fang, P. J. Frost, A. Iida-Klein, and T. J. Hahn, "Effects of nicotine on cellular function in UMR 106-01 osteoblast-like cells," Bone, vol. 12, no. 4, pp. 283-286, 1991.

[42] R. Chapurlat, "Epidemiology of osteoporosis," Journal Society Biology, vol. 202, no. 4, pp. 251-255, 2008.

[43] D. Kapoor and T. H. Jones, "Smoking and hormones in health and endocrine disorders," European Journal of Endocrinology, vol. 152, no. 4, pp. 491-499, 2005.

[44] H. Hermizi, O. Faizah, S. Ima-Nirwana, S. A. Nazrun, and M. Norazlina, "Beneficial effects of tocotrienol and tocopherol on bone histomorphometric parameters in sprague-dawley male rats after nicotine cessation," Calcified Tissue International, vol. 84, no. 1, pp. 65-74, 2009.

[45] C. R. Kessenich, "Calcium and vitamin D supplementation for postmenopausal bone health," Journal for Nurse Practitioners, vol. 3, no. 3, pp. 155-159, 2007.

[46] T. Imai, M. Omoto, K. Seki, and T. Harada, "The effects of long-term intake of restricted calcium, vitamin $\mathrm{D}$, and vitamin $\mathrm{E}$ and cadmium-added diets on various organs and bones of mice: a histological and the roentgenological study," Japanese Journal of Hygiene, vol. 50, no. 2, pp. 660-682, 1995.

[47] I. N. Sergeev, I. P. Arkhapchev, and V. B. Spirichev, "The role of vitamin $\mathrm{E}$ in the metabolism and reception of vitamin D," Biokhimiya, vol. 55, no. 11, pp. 1989-1995, 1990.

[48] M. Norazlina, S. Ima-Nirwana, M. T. A. Gapor, and B. A. K. Khalid, "Tocotrienols are needed for normal bone calcification in growing female rats," Asia Pacific Journal of Clinical Nutrition, vol. 11, no. 3, pp. 194-199, 2002.

[49] M. Norazlina, H. L. R. Ling, and S. Ima-Nirwana, "The effects of vitamin $\mathrm{E}$ or calcium supplementation on bone mineral composition in vitamin E deficient rats," Malaysian Journal of Biochemistry and Molecular Biology, vol. 7, pp. 1-5, 2002.

[50] H. Bismar, I. Diel, R. Ziegler, and J. Pfeilschifter, "Increased cytokine secretion by human bone marrow cells after menopause or discontinuation of estrogen replacement," Journal of Clinical Endocrinology and Metabolism, vol. 80, no. 11, pp. 3351-3355, 1995.

[51] M. E. Cohen-Solal, A. M. Graulet, M. A. Denne, J. Gueris, D. Baylink, and M. C. de Vernejoul, "Peripheral monocyte culture supernatants of menopausal women can induce bone resorption: involvement of cytokines," Journal of Clinical Endocrinology and Metabolism, vol. 77, no. 6, pp. 1648-1653, 1993.

[52] R. Pacifici, C. Brown, E. Puscheck et al., "Effect of surgical menopause and estrogen replacement on cytokine release from human blood mononuclear cells," Proceedings of the National Academy of Sciences of the United States of America, vol. 88, no. 12, pp. 5134-5138, 1991.
[53] M. C. Horowitz, "Cytokines and estrogen in bone: antiosteoporotic effects," Science, vol. 260, no. 5108, pp. 626-627, 1993.

[54] S. Ima-Nirwana and S. Suhaniza, "Effects of tocopherols and tocotrienols on body composition and bone calcium content in adrenalectomized rats replaced with dexamethasone," Journal of Medicinal Food, vol. 7, no. 1, pp. 45-51, 2004.

[55] B. J. Smith, E. A. Lucas, R. T. Turner et al., "Vitamin E provides protection for bone in mature hindlimb unloaded male rats," Calcified Tissue International, vol. 76, no. 4, pp. 272-279, 2005.

[56] S. C. Chai, C. I. Wei, K. B. Smith, and B. H. Arjmandi, "The role of vitamin E in reversing bone loss," Aging Clinical and Experimental Research, vol. 20, no. 6, pp. 521-527, 2008.

[57] A. N. Shuid, Z. Mehat, N. Mohamed, N. Muhammad, and I. N. Soelaiman, "Vitamin E exhibits bone anabolic actions in normal male rats," Journal of Bone and Mineral Metabolism, vol. 28, no. 2, pp. 149-156, 2010.

[58] M. Z. Mehat, A. N. Shuid, N. Mohamed, N. Muhammad, and I. N. Soelaiman, "Beneficial effects of vitamin e isomer supplementation on static and dynamic bone histomorphometry parameters in normal male rats," Journal of Bone and Mineral Metabolism, vol. 28, no. 5, pp. 503-509, 2010.

[59] D. Maggio, M. Barabani, M. Pierandrei et al., "Marked decrease in plasma antioxidants in aged osteoporotic women: results of a cross-sectional study," Journal of Clinical Endocrinology and Metabolism, vol. 88, no. 4, pp. 1523-1527, 2003.

[60] H. M. Macdonald, S. A. New, M. H. N. Golden, M. K. Campbell, and D. M. Reid, "Nutritional associations with bone loss during the menopausal transition: evidence of a beneficial effect of calcium, alcohol, and fruit and vegetable nutrients and of a detrimental effect of fatty acids," American Journal of Clinical Nutrition, vol. 79, no. 1, pp. 155-165, 2004.

[61] R. L. Wolf, J. A. Cauley, M. Pettinger et al., "Lack of a relation between vitamin and mineral antioxidants and bone mineral density: results from the women's health initiative," American Journal of Clinical Nutrition, vol. 82, no. 3, pp. 581-588, 2005.

[62] P. D. Delmas, "Biochemical markers for the assessment of bone turnover," in Osteoporosis: Etiology, Diagnosis and Management, pp. 319-333, Lippincott-Raven, Philadelphia, Pa, USA, 2nd edition, 1995. 


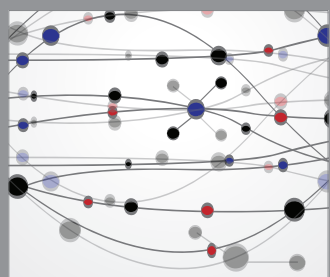

The Scientific World Journal
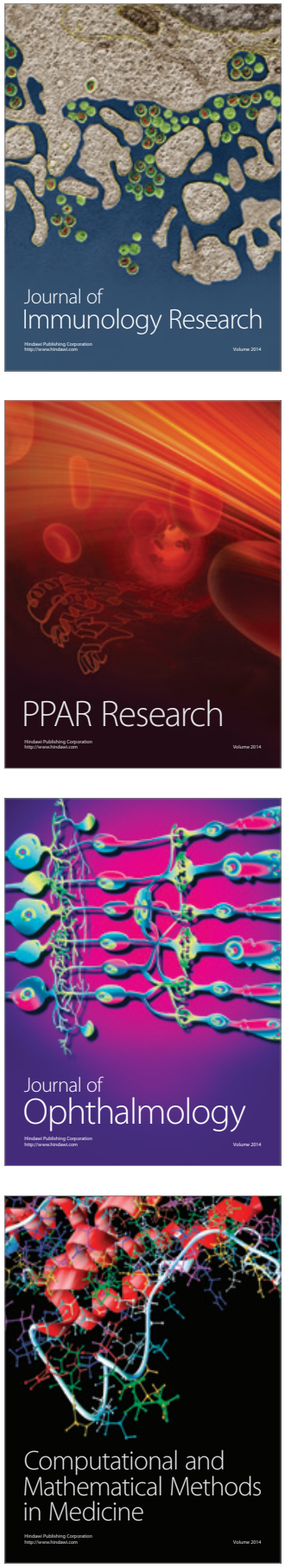

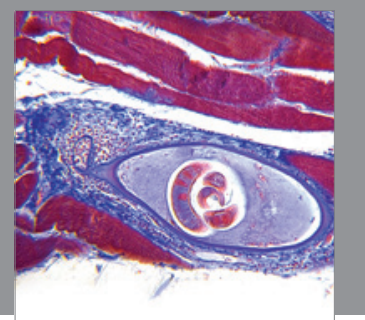

Gastroenterology

Research and Practice
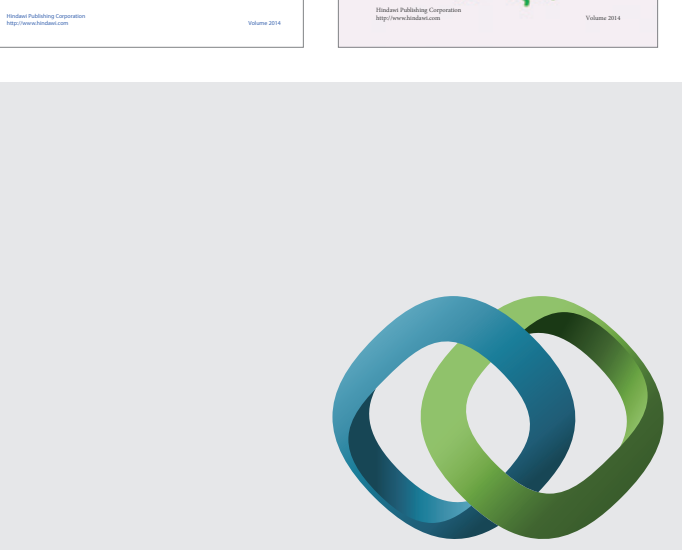

\section{Hindawi}

Submit your manuscripts at

http://www.hindawi.com
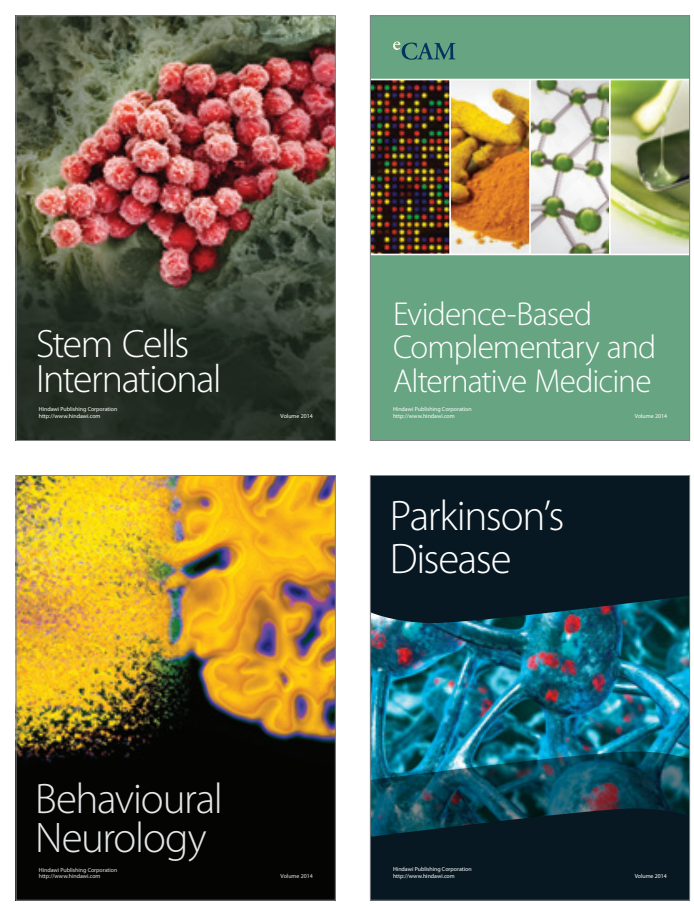

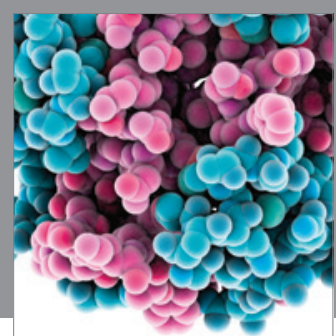

Journal of
Diabetes Research

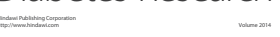

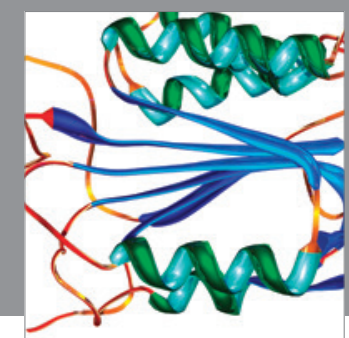

Disease Markers
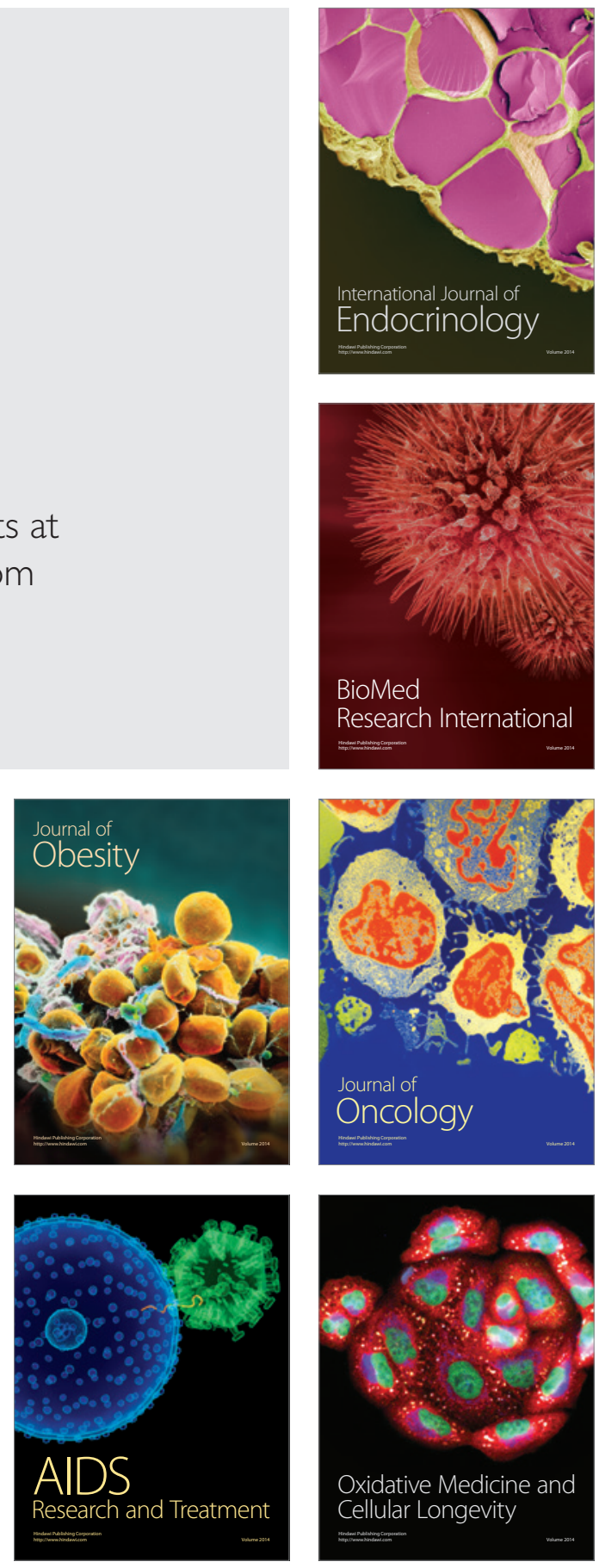